\title{
Adolescence as a unique developmental period
}

\author{
Natalia Jaworska, PhD; Glenda MacQueen, MD, PhD
}

Gaps exist between our understanding of adolescent brain development and clinical research involving the treatment of adolescents with emerging or established psychiatric disorders. In this editorial, we suggest that while basic research on brain development during adolescence appears to be thriving, our understanding of how to approach this developmental window from a clinical and policy perspective is lagging.

\section{Defining adolescence: How does it challenge clinical approaches?}

In the broadest sense, adolescence refers to the period marking the transition from childhood to adulthood. Historically, this typically spans from 12 to 18 years of age, which roughly corresponds to the time from pubertal onset (i.e., specific hormonal changes) to guardian independence (i.e., the legal definition of "adulthood" in many countries ${ }^{1}$ ). Adolescence frequently co-occurs with puberty, a biological phenomenon defined by a constellation of events that are driven by increases in adrenal and gonadal hormones, including the development of secondary sex characteristics and modulations in muscle and fat. ${ }^{2}$ It is associated with a period of increased risk-taking behaviours as well as increased emotional reactivity. ${ }^{3}$ This is typically coincident with changes in the social and school environment, such as spending less time with parents and more with peers, as well as an increase in autonomy. These behavioural changes occur in the context of developmental changes that are influenced by both external environmental and internal factors that elicit and reinforce behaviours. Adolescence is temporally confined but not fixed; as Casey and colleagues ${ }^{3}$ have suggested, it should be conceptualized as a developmental period rather than a temporal snapshot as it is highly variable behaviourally and developmentally.

Recent work has expanded the definition and timeframe of adolescence to include young adulthood, often up to about 25 years of age. While this encompasses some of the neural changes that occur beyond 18 years of age, it creates challenges in the clinical approaches to adolescents and the policies that guide them. It also exacerbates the challenges of both studying and treating young people throughout this time, as the brains, behavioural profiles and social demands and roles of a typically developing 12-year-old and those of a 24-year-old are strikingly different. The expanded definition of adolescence is, however, consistent with both a biological and sociological phenomenon known as the prolongation of adolescence. This refers to earlier pubertal onset, particularly in girls. ${ }^{1,4}$ Similarly, in terms of the social/personal responsibility associated with adult roles, adolescence has extended into the early 20s, with more individuals delaying traditional adult responsibilities (e.g., starting a family or full-time employment, buying property) in contemporary societies.

\section{Understanding adolescent brain development}

Many species are characterized by a developmental and behavioural transition from childhood (parental/conspecific dependence) to full maturity and behavioural independence. Animal studies are pivotal in helping us to understand the neural substrates associated with adolescence. The manipulation of domains, such as genetics, that allow us to gain insight into maturational processes are feasible only in animal models. ${ }^{5}$ Neuroimaging studies, however, have also provided us with new understanding of human brain development and activity during adolescence. This work complements animal studies and further emphasizes the fact that adolescence is a unique period relative to both childhood and adulthood rather than a linear progression from one to the other.

Morphometric analyses generally indicate that cortical grey matter volume declines in many regions of the brain from childhood throughout adolescence; the slope of this decline varies depending on the region. The grey matter volume of the mid-dorsolateral frontal prefrontal cortex (DLPFC), for example, peaks in mid-adolescence, declines and then stabilizes in early adulthood. ${ }^{6}$ White matter volumes show a more consistent linear increase from childhood through adolescence, with maximum volumes evident in late adolescence/early adulthood.7 The decreases in grey matter during adolescence have often been interpreted as pruning of redundant synapses, ${ }^{8}$ although there is relatively limited evidence for this interpretation. ${ }^{6}$ Another means of thinking about the developmental trajectory in adolescents is that regions subserving higher-order cognitive functioning develop and mature later than "primary" function regions. Finally, the development of subcortical regions is also pronounced

Correspondence to: G. MacQueen, Department of Psychiatry and Behavioural Neurosciences, University of Calgary, 1403 29th St. NW, Calgary AB T2N 2T9; gmmacque@ucalgary.ca

DOI: $10.1503 /$ jpn. 150268 
during adolescence, although this maturational process receives relatively little study in comparison to cortical development of frontal regions. ${ }^{3}$

Morphometric and imaging analyses have also been linked with behavioural data in animal and human studies. Behaviourally, nonhuman adolescents tend to exhibit increased novelty seeking, peer interactions and consummatory and hedonic behaviours. ${ }^{2}$ Similarly, human adolescents make risky choices and exhibit greater emotional reactivity than either children or adults. ${ }^{3}$ A primary cognitive feature defining the progression throughout adolescence is the ability to control impulses and delay gratification in favour of goal-directed and more optimized outcomes and behaviours. ${ }^{3,4}$ Accumulating data indicates that adolescent behaviour is differentially biased in motivationally charged contexts compared with adult behaviour; correspondingly, sensitivity to rewards peaks during adolescence. ${ }^{9}$ In a recent longitudinal study, Braams and colleagues ${ }^{10}$ assessed a large sample of children and young adults (age 8-27 yr) while measuring brain activity in response to rewards. Their work confirmed a longitudinal peak in nucleus accumbens (NAcc) activity in response to rewards in 15-17-year-old youths, but also demonstrated that NAcc activity was associated with individual difference measures in the drive for rewards. Pubertal development was linearly related to NAcc activity in response to rewards. ${ }^{10}$ Finally, sensation seeking has been shown to follow a curvilinear trajectory that peaks at age 10-15 years, while impulsivity follows a more linear pattern, decreasing with age.,9

Hare and colleagues ${ }^{11}$ found that adolescents exhibited initially exaggerated amygdala activity in response to fearful facial expressions relative to children and adults; this exaggerated activity was age-dependent, following a curvilinear pattern consistent with heightened emotional reactivity in adolescents. The extent to which this exaggerated response diminished over time was associated with anxiety measures in the youth. This suggests that although exaggerated emotional reactivity is evident in adolescence, the maladaptive pattern of nondiminished amygdala activity is associated with high levels of anxiety. Further, enhanced amygdala activity in response to fearful faces was inversely correlated with prefrontal cortex (PFC) activity, confirming a modulatory/control role by higher PFC centres on the amygdala. ${ }^{11}$

Van Duijvenvoorde and colleagues ${ }^{12}$ reported that learning rate was associated with intrinsic connectivity between the DLPFC and subcortical regions; this association increased over time with increasing age in 8-25-year-olds. Simultaneously, decreased connectivity between the DLPFC and motor areas was associated with better cognition and increased age, which may be related to increasing motor inhibition and impulse control with age. ${ }^{12}$ Further, an age-related increase in functional connectivity with the NAcc and other subcortical structures (hippocampus and caudate), the insula and more dorsal aspects of the anterior cingulate cortex were apparent. There are, therefore, differences in adolescent brain structure and connectivity that correspond with differences in emotional and cognitive function and that make adolescent brains distinct from those of children and adults.

\section{Wanted: policy and clinical approaches tailored to treating mental illness in adolescents}

Adolescence is also a distinct developmental period during which the incidence of many psychiatric illnesses rises dramatically; according to the National Comorbidity Survey Replication, three-quarters of psychiatric illnesses will onset by age 24 years. ${ }^{13}$ The diagnostic criteria for mental illnesses in adults are generally applied to adolescents, though the validity of this practice is questionable. Further, the treatment of various psychiatric symptoms in adolescents is often extrapolated from treatment approaches used in adults. For example, the treatment of adolescent depression and anxiety is based almost entirely on what is known from adult studies, in which the average age of patients is well beyond that of adolescents. Treatment studies of adolescents typically include youth aged 12-18 years. ${ }^{14,15}$ In large clinical trials of adults with depression, the portion of patients aged 18-25 years is often low; in the Sequenced Treatment Alternatives to Relieve Depression $\left(\mathrm{STAR}^{*} \mathrm{D}\right)$ trial, this age group comprised only $12 \%$ of the sample. Further, the average age of first onset of depression for the entire sample was older than 25 years. ${ }^{16}$ Older adolescents and those who treat them are consequently left with virtually no information on treatment strategies for depression specific to this developmental stage.

There is a need for further clinical research to inform treatment of young people with psychiatric illness. There is also a need for developmental science to inform policies that are relevant to youth, for example in approaches to substance use and abuse. Preclinical studies on alcohol, nicotine and cannabis, among other substances, suggest that the adolescent brain has differential sensitivity and response to these commonly used substances. ${ }^{17-19}$ Policies focused on regulation of these substances do not appear to consistently consider the fact that exposure of the adolescent brain to such substances may have long-lasting effects in ways that are not apparent in adults. As Hill noted in a 2014 editorial in this journal, ${ }^{20}$ the distribution of evidence-based documents to policy makers is critical in guiding and influencing policy decisions regarding substance use and regulation.

Adolescence represents a period of strength and resilience, yet psychiatric illness often begins during this developmental timeframe. Despite the increased number of studies highlighting that adolescence is a distinct period of neural development with different brain sensitivity and responsivity, few clinical or policy approaches have been tailored to adolescents. The developmental work highlighting that adolescence is a distinct developmental period should encourage further clinical research specific to adolescents.

Affiliations: From the Department of Psychiatry, McGill University, Montreal, Que. (Jaworska); and the Department of Psychiatry and Behavioural Neurosciences, University of Calgary, Calgary, Alta. (MacQueen), Canada.

Competing interests: None declared by N. Jaworska. G. MacQueen declares that she has received consultancy fees from Pfizer, Lundbeck, BMS, Servier, AstraZeneca and Lilly, lecture fees from the Norlien Foundation, and both lecture fees and payment for development of educational presentations from the Canadian Psychiatric Association. 


\section{References}

1. Dahl RE. Adolescent brain development: a period of vulnerabilities and opportunities. Keynote address. Ann N Y Acad Sci 2004;1021: $1-22$.

2. Spear LP. The adolescent brain and age-related behavioral manifestations. Neurosci Biobehav Rev 2000;24:417-63.

3. Casey BJ, Jones RM, Hare TA. The adolescent brain. Ann N Y Acad Sci 2008;1124:111-26.

4. Casey BJ, Duhoux S, Malter Cohen M. Adolescence: What do transmission, transition, and translation have to do with it? Neuron 2010;67:749-60.

5. Stevens HE, Vaccarino FM. How animal models inform child and adolescent psychiatry. J Am Acad Child Adolesc Psychiatry 2015; 54:352-9.

6. Paus T, Keshavan M, Giedd JN. Why do many psychiatric disorders emerge during adolescence? Nat Rev Neurosci 2008;9:947-57.

7. Pfefferbaum A, Mathalon DH, Sullivan EV, et al. A quantitative magnetic resonance imaging study of changes in brain morphology from infancy to late adulthood. Arch Neurol 1994;51:874-87.

8. Huttenlocher PR, Dabholkar AS. Regional differences in synaptogenesis in human cerebral cortex. J Comp Neurol 1997;387:167-78.

9. Cauffman E, Shulman EP, Steinberg L, et al. Age differences in affective decision making as indexed by performance on the Iowa Gambling Task. Dev Psychol 2010;46:193-207.

10. Braams BR, Van Duijvenvoorde AC, Peper JS, et al. Longitudinal changes in adolescent risk-taking: a comprehensive study of neural responses to rewards, pubertal development, and risk-taking behavior. J Neurosci 2015;35:7226-38.

11. Hare TA, Tottenham N, Galvan A, et al. Biological substrates of emotional reactivity and regulation in adolescence during an emotional go-nogo task. Biol Psychiatry 2008;63:927-34.

12. Van Duijvenvoorde AC, Achterberg M, Braams BR, et al. Testing a dual-systems model of adolescent brain development using resting-state connectivity analyses. Neuroimage 2015.

13. Kessler RC, Berglund P, Demler O, et al. Lifetime prevalence and age-of-onset distributions of DSM-IV disorders in the National Comorbidity Survey Replication. Arch Gen Psychiatry 2005;62:593-602.

14. Rush AJ, Wisniewski SR, Warden D, et al. Selecting among second-step antidepressant medication monotherapies: predictive value of clinical, demographic, or first-step treatment features. Arch Gen Psychiatry 2008;65:870-80.

15. March J, Silva S, Petrycki S, et al. Fluoxetine, cognitive-behavioral therapy, and their combination for adolescents with depression: treatment for adolescents with depression study (TADS) randomized controlled trial. JAMA 2004;292:807-20.

16. Trivedi MH, Fava M, Wisniewski SR, et al. Medication augmentation after the failure of SSRIs for depression. N Engl J Med 2006;354:1243-52.

17. White AM, Swartzwelder HS. Hippocampal function during adolescence: a unique target of ethanol effects. Ann N Y Acad Sci 2004; 1021:206-20.

18. Smith RF, McDonald CG, Bergstrom HC, et al. Adolescent nicotine induces persisting changes in development of neural connectivity. Neurosci Biobehav Rev 2015;55:432-43.

19. Higuera-Matas A, Ucha M, Ambrosio E. Long-term consequences of perinatal and adolescent cannabinoid exposure on neural and psychological processes. Neurosci Biobehav Rev 2015;55:119-46.

20. Hill MN. Clearing the smoke: What do we know about adolescent cannabis use and schizophrenia? J Psychiatry Neurosci 2014;39:75-7.

\section{$J P N^{\prime} s$ top viewed articles*}

1. $\quad N$-acetylcysteine in psychiatry: current therapeutic evidence and potential mechanisms of action

Dean et al.

J Psychiatry Neurosci 2011;36(2):78-86

2. What is the best treatment for bipolar depression? Young

J Psychiatry Neurosci 2008;33(6):487-8

3. On the neurobiology of hallucinations

Boksa

J Psychiatry Neurosci 2009;34(4):260-2

4. Premenstrual dysphoric disorder: burden of illness and treatment update

Pearlstein and Steiner

J Psychiatry Neurosci 2008;33(4):291-301

5. Escitalopram and QTc prolongation

Hasnain

J Psychiatry Neurosci 2013;38(4):E11

6. Biologic effects of mindfulness meditation: growing insights into neurobiologic aspects of the prevention of depression

Young

J Psychiatry Neurosci 2011;36(2):75-7

7. Fish oils for depression?

Young

J Psychiatry Neurosci 2008;33(1):80

8. Effects of sustained serotonin reuptake inhibition on the firing of dopamine neurons in the rat ventral tegmental area

Dremencov et al.

J Psychiatry Neurosci 2009;34(3):223-9

\footnotetext{
* Based on page views on PubMed Central of content published in the last 7 years.

Updated Aug. 7, 2015.
} 